\title{
GEOMETRIC STABILITY SWITCH CRITERIA IN DELAY DIFFERENTIAL SYSTEMS WITH DELAY DEPENDENT PARAMETERS*
}

\author{
EDOARDO BERETTA ${ }^{\dagger}$ AND YANG KUANG B $^{\ddagger}$
}

\begin{abstract}
In most applications of delay differential equations in population dynamics, the need of incorporation of time delays is often the result of the existence of some stage structure. Since the through-stage survival rate is often a function of time delays, it is easy to conceive that these models may involve some delay dependent parameters. The presence of such parameters often greatly complicates the task of an analytical study of such models. The main objective of this paper is to provide practical guidelines that combine graphical information with analytical work to effectively study the local stability of some models involving delay dependent parameters. Specifically, we shall show that the stability of a given steady state is simply determined by the graphs of some functions of $\tau$ which can be expressed explicitly and thus can be easily depicted by Maple and other popular software. In fact, for most application problems, we need only look at one such function and locate its zeros. This function often has only two zeros, providing thresholds for stability switches. The common scenario is that as time delay increases, stability changes from stable to unstable to stable, implying that a large delay can be stabilizing. This scenario often contradicts the one provided by similar models with only delay independent parameters.
\end{abstract}

Key words. delay differential equations, stability switch, characteristic equations, stage structure, population models

AMS subject classifications. 34K18, 34K20, 92D25

PII. S0036141000376086

1. Introduction. Due to the fact that actions and reactions take time to take effect in real-life problems, one often introduces time delays in the variables being modeled. This often yields delay differential and delay difference models [11], [21], [19]. Some of these models have delay dependent parameters (for example, [1], [2], [3], [4], [9], [10], [22]), while most of them contain only parameters that are independent of time delays.

In most applications of delay differential equations in population dynamics, the need of incorporation of a time delay is often the result of the existence of some stage structure [1], [3], [10], [11], [12], [15]. Indeed, just about every population goes through some distinct life stages [23], [18]. Since the through-stage survival rate is often a function of a time delay, it is thus easy to conceive that these models will inevitably involve some delay dependent parameters.

In view of the fact that it is often difficult to analytically study models with delay dependent parameters even if only a single discrete delay is present, it is natural to resort to the help of computer programs. The main objective of this paper is to provide practical guidelines that combine graphical information with analytical work to effectively study the local stability of models involving delay dependent parameters. To apply our results, one need only perform some routine computation (using our analytical criteria) and generate some simple graphs which can be easily produced by popular software such as Maple. The results also can be readily confirmed by some

\footnotetext{
*Received by the editors July 28, 2000; accepted for publication (in revised form) September 4, 2001; published electronically February 14, 2002.

http://www.siam.org/journals/sima/33-5/37608.html

${ }^{\dagger}$ Istituto di Biomatematica, Universita di Urbino, I-61029 Urbino, Italy (e.beretta@mat.uniurb.it).

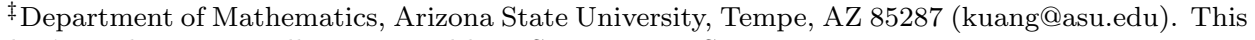
author's work was partially supported by NSF grant DMS-0077790.
} 
selective simulations using the freely available and user friendly software XPP. No other programming skill is required.

Specifically, we shall show that the stability of a given steady state is simply determined by the graphs of some functions of $\tau$ which can be expressed explicitly and thus can be easily depicted by Maple and other popular software. In fact, for most application problems, we need only look at one such function and locate its zeros. This function often has only two zeros, providing thresholds for stability switches. The common scenario is that as time delay increases, stability changes from stable to unstable to stable. We hope this work will show that it is important and possible to systematically study local stability aspects of some models with delay dependent parameters.

In the next section, we present a general geometric criterion that, theoretically speaking, can be applied to models with many delays, or even distributed delays [5], [7]. This is followed by a section dealing with the simple case of a first order characteristic equation, providing more user friendly geometric and analytic criteria for stability switches. In section 4 , we accomplish the same for the second order case. The analytical criteria provided for the first and second order cases can be used to obtain some insightful analytical statements and can be helpful for conducting simulations. Examples are provided for both first and second order cases to illustrate the applications of our criteria. A discussion section concludes the paper.

2. A general geometric criterion. In this section we study the occurrence of any possible stability switching resulting from the increase of value of the time delay $\tau$ for the general characteristic equation

$$
D(\lambda, \tau)=0 .
$$

Here

$$
D(\lambda, \tau)=P_{n}(\lambda, \tau)+Q_{m}(\lambda, \tau) e^{-\lambda \tau}
$$

and

$$
P_{n}(\lambda, \tau)=\sum_{k=0}^{n} p_{k}(\tau) \lambda^{k}, \quad Q_{m}(\lambda, \tau)=\sum_{k=0}^{m} q_{k}(\tau) \lambda^{k} .
$$

In (2.3), $n, m \in \mathbf{N}_{0}, n>m$, and $p_{k}(\cdot), q_{k}(\cdot): \mathbf{R}_{+0} \rightarrow \mathbf{R}$ are continuous and differentiable functions of $\tau$ such that

$$
P_{n}(0, \tau)+Q_{m}(0, \tau)=p_{0}(\tau)+q_{0}(\tau) \neq 0 \quad \forall \tau \in \mathbf{R}_{+0},
$$

i.e., $\lambda=0$ is not a characteristic root of (2.1).

In the following "- " denotes complex and conjugate. $P_{n}(\lambda, \tau), Q_{m}(\lambda, \tau)$ are analytic functions in $\lambda$ and differentiable in $\tau$ for which we assume ([21, p. 83]; see also [8] and [6]) the following:

(i) If $\lambda=i \omega, \omega \in \mathbf{R}$, then $P_{n}(i \omega, \tau)+Q_{m}(i \omega, \tau) \neq 0, \tau \in \mathbf{R}$;

(ii) $\lim \sup \left\{\left|Q_{m}(\lambda, \tau) / P_{n}(\lambda, \tau)\right|:|\lambda| \rightarrow \infty, \operatorname{Re} \lambda \geq 0\right\}<1$ for any $\tau$;

(iii) $F(\omega, \tau):=\left|P_{n}(i \omega, \tau)\right|^{2}-\left|Q_{m}(i \omega, \tau)\right|^{2}$ for each $\tau$ has at most a finite number of real zeros.

(iv) Each positive root $\omega(\tau)$ of $F(\omega, \tau)=0$ is continuous and differentiable in $\tau$ whenever it exists. 
Assumption (i) implies that $P_{n}(\lambda, \tau)$ and $Q_{m}(\lambda, \tau)$ have no common imaginary roots. This is needed to ensure that threshold time delays can be explicitly expressed (see the comment after (2.10)). Assumption (ii) is needed to ensure that there are no roots bifurcating from infinity. Assumption (iii) is needed to ensure that there are only finite "gates" for roots to cross the imaginary axis for any given $\tau$. Assumption (iv) is needed to compute the derivative of the imaginary roots with respect to $\tau$. We remark also that, since $P_{n}, Q_{m}$ have real coefficients, then

$$
\overline{P_{n}(-i \omega, \tau)}=P_{n}(i \omega, \tau), \quad \overline{Q_{m}(-i \omega, \tau)}=Q_{m}(i \omega, \tau)
$$

for each $\tau$ and any real $\omega$, thus ensuring that if $\lambda=i \omega$ for some real $\omega$ is a characteristic root of (2.1), then also $\lambda=-i \omega$ is a characteristic root. In the following, we will drop the indices $n, m$ from $P_{n}, Q_{m}$. Furthermore, we will denote by $P_{R}, Q_{R}$ the real parts of $P$ and $Q$, respectively, and by $P_{I}, Q_{I}$ the imaginary parts of $P$ and $Q$, respectively. Hence, we can write

$$
P(\lambda, \tau)=P_{R}(\lambda, \tau)+i P_{I}(\lambda, \tau), \quad Q(\lambda, \tau)=Q_{R}(\lambda, \tau)+i Q_{I}(\lambda, \tau),
$$

where $P_{R}, P_{I}, Q_{R}, Q_{I}$ are real functions. In the following we will use this nomenclature for derivatives. The total derivative, say of $P(\lambda, \tau)$, with respect to $\tau$ will be denoted by

$$
D_{\tau} P(\lambda, \tau):=P_{\lambda}^{\prime}(\lambda, \tau) \frac{d \lambda}{d \tau}+P_{\tau}^{\prime}(\lambda, \tau),
$$

where $P_{\lambda}^{\prime}(\lambda, \tau):=\partial_{\lambda} P(\lambda, \tau), P_{\tau}^{\prime}(\lambda, \tau):=\partial_{\tau} P(\lambda, \tau)$ are the partial derivatives with respect to $\lambda, \tau$, respectively. Of course, after the partial derivation of $P(\lambda, \tau)$ with respect to $\lambda$, its real part can be separated from its imaginary one. We then have

$$
P_{\lambda}^{\prime}(\lambda, \tau)=P_{R_{\lambda}}^{\prime}(\lambda, \tau)+i P_{I_{\lambda}}^{\prime}(\lambda, \tau)
$$

and the same nomenclature applies for derivatives of $Q(\lambda, \tau)$. Similarly, $F_{\omega}^{\prime}(\omega, \tau)=$ $\partial_{\omega} F(\omega, \tau)$ denotes the partial derivative of $F(\omega, \tau)$ with respect to $\omega$, and so on. This stated, let us consider the general problem. Since for increasing $\tau$ the imaginary axis cannot be crossed by $\lambda(\tau)=0$ for some $\tau>0$ (see (2.4)), we look for the occurrence of a pair of simple and conjugate imaginary roots $\lambda= \pm i \omega(\tau), \omega(\tau)$ real and positive, which crosses the imaginary axis at some positive $\tau$ value, say $\tau^{*}$. Because of (2.5), without loss of generality, we can consider just $\lambda=i \omega(\tau), \omega(\tau)>0$, and the possibility that it is a root of the characteristic equation $(2.1)$. Then $\omega(\tau)$ must satisfy the following:

$$
\left\{\begin{array}{l}
Q_{I}(i \omega, \tau) \sin \omega \tau+Q_{R}(i \omega, \tau) \cos \omega \tau=-P_{R}(i \omega, \tau) \\
-Q_{R}(i \omega, \tau) \sin \omega \tau+Q_{I}(i \omega, \tau) \cos \omega \tau=-P_{I}(i \omega, \tau)
\end{array}\right.
$$

which gives

$$
\left\{\begin{aligned}
\sin \omega \tau & =\frac{-P_{R}(i \omega, \tau) Q_{I}(i \omega, \tau)+P_{I}(i \omega, \tau) Q_{R}(i \omega, \tau)}{|Q(i \omega, \tau)|^{2}} \\
\cos \omega \tau & =-\frac{P_{R}(i \omega, \tau) Q_{R}(i \omega, \tau)+P_{I}(i \omega, \tau) Q_{I}(i \omega, \tau)}{|Q(i \omega, \tau)|^{2}}
\end{aligned}\right.
$$

where $|Q(i \omega, \tau)|^{2} \neq 0$ because of assumption (i) (since $D(i \omega, \tau)=Q(i \omega, \tau)=0$ together imply $P(i \omega, \tau)=0)$. 
On the other hand, since (2.10) can be written as

$$
\sin \omega \tau=\operatorname{Im}\left(\frac{P(i \omega, \tau)}{Q(i \omega, \tau)}\right), \quad \cos \omega \tau=-\operatorname{Re}\left(\frac{P(i \omega, \tau)}{Q(i \omega, \tau)}\right),
$$

if $\omega$ satisfies (2.10), then $\omega(\tau)$ must satisfy that

$$
|P(i \omega, \tau)|^{2}=|Q(i \omega, \tau)|^{2}
$$

i.e., $\omega(\tau)$ must be a (positive) root of

$$
F(\omega, \tau):=|P(i \omega, \tau)|^{2}-|Q(i \omega, \tau)|^{2} .
$$

Assume that $I \subseteq \mathbf{R}_{+0}$ is the set where $\omega(\tau)$ is a positive root of (2.12) and for $\tau \notin I$, $\omega(\tau)$ is not definite. Then for all $\tau$ in $I, \omega(\tau)$ satisfies that

$$
F(\omega, \tau)=0 .
$$

Hence, differentiating (2.13) with respect to $\tau$ we get

$$
F_{\omega}^{\prime}(\omega, \tau) \omega^{\prime}+F_{\tau}^{\prime}(\omega, \tau)=0, \quad \tau \in I,
$$

where

$$
\left\{\begin{array}{l}
F_{\omega}^{\prime}=2\left[\left(P_{R_{\omega}}^{\prime} P_{R}+P_{I_{\omega}}^{\prime} P_{I}\right)-\left(Q_{R_{\omega}}^{\prime} Q_{R}+Q_{I_{\omega}}^{\prime} Q_{I}\right)\right] \\
F_{\tau}^{\prime}=2\left[\left(P_{R_{\tau}}^{\prime} P_{R}+P_{I_{\tau}}^{\prime} P_{I}\right)-\left(Q_{R_{\tau}}^{\prime} Q_{R}+Q_{I_{\tau}}^{\prime} Q_{I}\right)\right]
\end{array}\right.
$$

Now it is important to notice that if $\tau \notin I$, then there are no positive $\omega(\tau)$ solutions of (2.13), and we cannot have stability switches. Furthermore, for any $\tau \in I$ where $\omega(\tau)$ is a positive solution of (2.13), we can define the angle $\theta(\tau) \in[0,2 \pi]$ as the solution of $(2.10)$ :

$$
\left\{\begin{array}{l}
\sin \theta(\tau)=\frac{-P_{R}(i \omega, \tau) Q_{I}(i \omega, \tau)+P_{I}(i \omega, \tau) Q_{R}(i \omega, \tau)}{|Q(i \omega, \tau)|^{2}} \\
\cos \theta(\tau)=-\frac{P_{R}(i \omega, \tau) Q_{R}(i \omega, \tau)+P_{I}(i \omega, \tau) Q_{I}(i \omega, \tau)}{|Q(i \omega, \tau)|^{2}}
\end{array}\right.
$$

and the relation between the arguments " $\theta(\tau)$ " in $(2.16)$ and " $\omega(\tau) \tau$ " in (2.10) for $\tau \in I$ must be

$$
\omega(\tau) \tau=\theta(\tau)+n 2 \pi, \quad n \in \mathbf{N}_{0} .
$$

Hence, we can define the maps $\tau_{n}: I \rightarrow \mathbf{R}_{+0}$ given by

$$
\tau_{n}(\tau):=\frac{\theta(\tau)+n 2 \pi}{\omega(\tau)}, \quad n \in \mathbf{N}_{0}, \quad \tau \in I,
$$

where $\omega(\tau)$ is a positive root of (2.13). Let us introduce the functions $I \rightarrow \mathbf{R}$,

$$
S_{n}(\tau):=\tau-\tau_{n}(\tau), \quad \tau \in I, \quad n \in \mathbf{N}_{0},
$$

that are continuous and differentiable in $\tau$ as shown in the following lemma.

Lemma 2.1. Assume that $\omega(\tau)$ is a positive real root of (2.13) defined for $\tau \in I$, which is continuous and differentiable. Assume further that (i) holds true. Then the functions $S_{n}(\tau), n \in \mathbf{N}_{0}$, are continuous and differentiable on $I$. 
Proof. Assume that $\theta(\tau)$ is, for example, a monotone increasing function in a neighborhood $I_{\delta}\left(\tau^{\prime}\right)$ of $\tau^{\prime} \in I$, where $\theta\left(\tau^{\prime}\right)=2 \pi$. Since $\theta(\tau)$ must belong to $[0,2 \pi]$ at $\tau^{\prime}, \theta(\tau)$ may have a jump of height $2 \pi$ down to 0 at $\tau^{\prime}$. This will give rise to the first kind of discontinuity for $\tau_{n}(\tau)$ and $S_{n}(\tau)$ with a jump of height $2 \pi / \omega\left(\tau^{\prime}\right)$ at $\tau^{\prime}$. Without such a discontinuity, Remark 4.1 (see below) implies that $\theta(\tau)$ is continuous and differentiable on $I$ and so are $\tau_{n}(\tau)$ and $S_{n}(\tau)$. Thus it is enough to prove that $\theta(\tau) \neq 0,2 \pi$ on $I$, and hence $\theta(\tau) \in(0,2 \pi)$ on $I$. Assumption (i) implies that either

(a) $P_{R}(i \omega, \tau)+Q_{R}(i \omega, \tau) \neq 0$, or

(b) $P_{I}(i \omega, \tau)+Q_{I}(i \omega, \tau) \neq 0$.

Assume first that (a) holds true. Then either

(a1) $P_{R}(i \omega, \tau) \neq 0$, or

(a2) $Q_{R}(i \omega, \tau) \neq 0$.

Assume now that (a1) is true. If $\theta(\tau)=0,2 \pi$, then $\sin \theta(\tau)=0$ and $\cos \theta(\tau)=1$. From the first of (4.16), we have

$$
Q_{I}=P_{I} Q_{R} / P_{R}
$$

Substituting this into the second equation of (4.16) yields (since $\omega$ is a root of $F(\omega, \tau)=0)$

$$
\cos \theta(\tau)=-Q_{R} / P_{R}
$$

Hence $\cos \theta(\tau)=1$ implies that $P_{R}+Q_{R}=0$, contradicting (a). The proof for case (a2) is similar and so is the proof for (b). This proves the lemma.

We can also prove the following theorem.

TheOrem 2.2. Assume that $\omega(\tau)$ is a positive real root of (2.13) defined for $\tau \in I, I \subseteq \mathbf{R}_{+0}$, and at some $\tau^{*} \in I$,

$$
S_{n}\left(\tau^{*}\right)=0 \quad \text { for some } n \in \mathbf{N}_{0} .
$$

Then a pair of simple conjugate pure imaginary roots $\lambda_{+}\left(\tau^{*}\right)=i \omega\left(\tau^{*}\right), \lambda_{-}\left(\tau^{*}\right)=$ $-i \omega\left(\tau^{*}\right)$ of $(2.1)$ exists at $\tau=\tau^{*}$ which crosses the imaginary axis from left to right if $\delta\left(\tau^{*}\right)>0$ and crosses the imaginary axis from right to left if $\delta\left(\tau^{*}\right)<0$, where

$$
\delta\left(\tau^{*}\right)=\operatorname{sign}\left\{\left.\frac{d \operatorname{Re} \lambda}{d \tau}\right|_{\lambda=i \omega\left(\tau^{*}\right)}\right\}=\operatorname{sign}\left\{F_{\omega}^{\prime}\left(\omega\left(\tau^{*}\right), \tau^{*}\right)\right\} \operatorname{sign}\left\{\left.\frac{d S_{n}(\tau)}{d \tau}\right|_{\tau=\tau^{*}}\right\} .
$$

Proof. The existence part of the theorem follows from the requirement (2.17) which ensures that if and only if $\tau^{*} \in I$ is a zero of $S_{n}(\tau)$ for some $n \in \mathbf{N}_{0}, \lambda=$ $\pm i \omega\left(\tau^{*}\right)$ together with $\omega\left(\tau^{*}\right)>0$, a solution of (2.13), are characteristic roots of $(2.1)$.

To prove the geometric criterion (2.21) we remark that

$$
\operatorname{sign}\left\{\frac{d \operatorname{Re} \lambda}{d \tau}\right\}=\operatorname{sign}\left\{\operatorname{Re}\left(\frac{d \lambda}{d \tau}\right)^{-1}\right\} .
$$

Then differentiating (2.1) with respect to $\tau$ we obtain that

$$
\begin{aligned}
& \left(\frac{d \lambda}{d \tau}\right)\left[P_{\lambda}^{\prime}(\lambda, \tau)+\left(Q_{\lambda}^{\prime}(\lambda, \tau)-\tau Q(\lambda, \tau)\right) e^{-\lambda \tau}\right] \\
= & \lambda Q(\lambda, \tau) e^{-\lambda \tau}-\left[P_{\tau}^{\prime}(\lambda, \tau)+Q_{\tau}^{\prime}(\lambda, \tau) e^{-\lambda \tau}\right] .
\end{aligned}
$$


From (2.2), we have

$$
e^{\lambda \tau}=-\frac{Q(\lambda, \tau)}{P(\lambda, \tau)}
$$

Hence, we obtain

$$
\left(\frac{d \lambda}{d \tau}\right)^{-1}=\left(-\frac{P_{\lambda}^{\prime}(\lambda, \tau)}{P(\lambda, \tau)}+\frac{Q_{\lambda}^{\prime}(\lambda, \tau)}{Q(\lambda, \tau)}-\tau\right) /\left(\lambda+\frac{P_{\tau}^{\prime}(\lambda, \tau)}{P(\lambda, \tau)}-\frac{Q_{\tau}^{\prime}(\lambda, \tau)}{Q(\lambda, \tau)}\right)
$$

where $P(i \omega, \tau), Q(i \omega, \tau) \neq 0$ due to assumption (i). Assume that $\lambda=i \omega(\tau)$, where $\omega(\tau)>0$ is a root of (2.13). Then from (2.24) we obtain

$$
\begin{aligned}
& \operatorname{sign}\left\{\left.\frac{d \operatorname{Re} \lambda}{d \tau}\right|_{\lambda=i \omega}\right\} \\
&(2.25)=\operatorname{sign} \operatorname{Re}\left\{\frac{-P_{\lambda}^{\prime}(i \omega, \tau) \overline{P(i \omega, \tau)}+Q_{\lambda}^{\prime}(i \omega, \tau) \overline{Q(i \omega, \tau)}-\tau|P(i \omega, \tau)|^{2}}{P_{\tau}^{\prime}(i \omega, \tau) \overline{P(i \omega, \tau)}-Q_{\tau}^{\prime}(i \omega, \tau) \overline{Q(i \omega, \tau)}+i \omega|P(i \omega, \tau)|^{2}}\right\} .
\end{aligned}
$$

Now we remark that

$$
i P_{\lambda}^{\prime}(i \omega, \tau)=P_{\omega}^{\prime}(i \omega, \tau), \quad i Q_{\lambda}^{\prime}(i \omega, \tau)=Q_{\omega}^{\prime}(i \omega, \tau)
$$

Hence, in (2.25) we have

$$
\begin{aligned}
& -P_{\lambda}^{\prime}(i \omega, \tau) \overline{P(i \omega, \tau)}+Q_{\lambda}^{\prime}(i \omega, \tau) \overline{Q(i \omega, \tau)} \\
= & i\left[\left(P_{R_{\omega}}^{\prime} P_{R}+P_{I_{\omega}}^{\prime} P_{I}\right)-\left(Q_{R_{\omega}}^{\prime} Q_{R}+Q_{I_{\omega}}^{\prime} Q_{I}\right)\right] \\
& -\left[\left(P_{I_{\omega}}^{\prime} P_{R}-P_{I} P_{R_{\omega}}^{\prime}\right)-\left(Q_{I_{\omega}}^{\prime} Q_{R}-Q_{I} Q_{R_{\omega}}^{\prime}\right)\right],
\end{aligned}
$$

which due to $(2.15)$ becomes

$$
\begin{aligned}
& -P_{\lambda}^{\prime}(i \omega, \tau) \overline{P(i \omega, \tau)}+Q_{\lambda}^{\prime}(i \omega, \tau) \overline{Q(i \omega, \tau)} \\
= & i \frac{F_{\omega}^{\prime}(\omega, \tau)}{2}-\left[\left(P_{R} P_{I_{\omega}}^{\prime}-P_{I} P_{R_{\omega}}^{\prime}\right)-\left(Q_{R} Q_{I_{\omega}}^{\prime}-Q_{I} Q_{R_{\omega}}^{\prime}\right)\right] .
\end{aligned}
$$

Similarly, in (2.25) we have

$$
\begin{aligned}
& P_{\tau}^{\prime}(i \omega, \tau) \overline{P(i \omega, \tau)}-Q_{\tau}^{\prime}(i \omega, \tau) \overline{Q(i \omega, \tau)} \\
= & \frac{1}{2} F_{\tau}^{\prime}(\omega, \tau)+i\left[\left(P_{R} P_{I_{\tau}}^{\prime}-P_{I} P_{R_{\tau}}^{\prime}\right)-\left(Q_{R} Q_{I_{\tau}}^{\prime}-Q_{I} Q_{R_{\tau}}^{\prime}\right)\right] .
\end{aligned}
$$

Furthermore, remember that from (2.14)

$$
F_{\tau}^{\prime}(\omega, \tau)=-F_{\omega}^{\prime}(\omega, \tau) \omega^{\prime}
$$

Hence, from (2.28)-(2.30) in (2.25) we obtain

$$
\operatorname{sign}\left\{\left.\frac{d \operatorname{Re} \lambda}{d \tau}\right|_{\lambda=i \omega}\right\}=\operatorname{sign} \operatorname{Re}\left\{\frac{-2\left\{U+\tau|P(i \omega, \tau)|^{2}\right\}+i F_{\omega}^{\prime}(\omega, \tau)}{F_{\tau}^{\prime}(\omega, \tau)+i 2\left\{V+\omega|P(i \omega, \tau)|^{2}\right\}}\right\},
$$

where

$$
U:=\left(P_{R} P_{I_{\omega}}^{\prime}-P_{I} P_{R_{\omega}}^{\prime}\right)-\left(Q_{R} Q_{I_{\omega}}^{\prime}-Q_{I} Q_{R_{\omega}}^{\prime}\right), \quad V:=\left(P_{R} P_{I_{\tau}}^{\prime}-P_{I} P_{R_{\tau}}^{\prime}\right)-\left(Q_{R} Q_{I_{\tau}}^{\prime}-Q_{I} Q_{R_{\tau}}^{\prime}\right)
$$


Simple computation yields

(2.31) $\operatorname{sign}\left\{\left.\frac{d \operatorname{Re} \lambda}{d \tau}\right|_{\lambda=i \omega}\right\}=\operatorname{sign}\left\{F_{\omega}^{\prime}(\omega, \tau)\right\} \operatorname{sign}\left\{\tau \omega^{\prime}+\omega+\frac{U \omega^{\prime}+V}{|P(i \omega, \tau)|^{2}}\right\}$.

Now observe that if $S_{n}\left(\tau^{*}\right)=0$, then $S_{n}^{\prime}\left(\tau^{*}\right)=\left(\omega\left(\tau^{*}\right)+\tau^{*} \omega^{\prime}\left(\tau^{*}\right)-\theta^{\prime}\left(\tau^{*}\right)\right) / \omega\left(\tau^{*}\right)$, which gives

$$
\operatorname{sign}\left\{S_{n}^{\prime}\left(\tau^{*}\right)\right\}=\operatorname{sign}\left\{\omega\left(\tau^{*}\right)+\tau^{*} \omega^{\prime}\left(\tau^{*}\right)-\theta^{\prime}\left(\tau^{*}\right)\right\},
$$

where $\theta^{\prime}\left(\tau^{*}\right)$ can be computed with the help of (2.16). Let

$$
\left\{\begin{array}{l}
\psi(\tau)=-P_{R}(i \omega, \tau) Q_{I}(i \omega, \tau)+P_{I}(i \omega, \tau) Q_{R}(i \omega, \tau) \\
\varphi(\tau)=P_{R}(i \omega, \tau) Q_{R}(i \omega, \tau)+P_{I}(i \omega, \tau) Q_{I}(i \omega, \tau)
\end{array}\right.
$$

then for all $\tau \in I, \theta^{\prime}(\tau)$ is defined as (see Remark 2.1)

$$
\theta^{\prime}(\tau)=\frac{\psi(\tau) \varphi^{\prime}(\tau)-\psi^{\prime}(\tau) \varphi(\tau)}{|P(i \omega, \tau)|^{4}}
$$

where

$$
\left\{\begin{array}{c}
\varphi^{\prime}(\tau)=\left(P_{R_{\omega}}^{\prime} Q_{R}+P_{R} Q_{R_{\omega}}^{\prime}+P_{I_{\omega}}^{\prime} Q_{I}+P_{I} Q_{I_{\omega}}^{\prime}\right) \omega^{\prime} \\
+\left(P_{R_{\tau}}^{\prime} Q_{R}+P_{R} Q_{R_{\tau}}^{\prime}+P_{I_{\tau}}^{\prime} Q_{I}+P_{I} Q_{I_{\tau}}^{\prime}\right) \\
\psi^{\prime}(\tau)=\left(-P_{R_{\omega}} Q_{I}-P_{R} Q_{I_{\omega}}^{\prime}+Q_{R_{\omega}}^{\prime} P_{I}+Q_{R} P_{I_{\omega}}^{\prime}\right) \omega^{\prime} \\
+\left(-P_{R_{\tau}}^{\prime} Q_{I}-P_{R} Q_{I_{\tau}}^{\prime}+Q_{R_{\tau}}^{\prime} P_{I}+Q_{R} P_{I_{\tau}}^{\prime}\right)
\end{array}\right.
$$

Hence, from (2.34), (2.35) we have

$$
\begin{aligned}
|P(i \omega, \tau)|^{4} \theta^{\prime}(\tau)=\omega^{\prime}\left\{\left(P_{R_{\omega}}^{\prime} Q_{R}+P_{R} Q_{R_{\omega}}^{\prime}+P_{I_{\omega}}^{\prime} Q_{I}+P_{I} Q_{I_{\omega}}^{\prime}\right)\left(-P_{R} Q_{I}+Q_{R} P_{I}\right)\right. & \\
& \left.\left(-P_{R_{\omega}}^{\prime} Q_{I}-P_{R} Q_{I_{\omega}}^{\prime}+Q_{R_{\omega}}^{\prime} P_{I}+Q_{R} P_{I_{\omega}}^{\prime}\right)\left(P_{R} Q_{R}+P_{I} Q_{I}\right)\right\} \\
& +\left(P_{R_{\tau}}^{\prime} Q_{R}+P_{R} Q_{R_{\tau}}^{\prime}+P_{I_{\tau}}^{\prime} Q_{I}+P_{I} Q_{I_{\tau}}^{\prime}\right)\left(-P_{R} Q_{I}+Q_{R} P_{I}\right) \\
& -\left(-P_{R_{\tau}}^{\prime} Q_{I}-P_{R} Q_{I_{\tau}}^{\prime}+Q_{R_{\tau}}^{\prime} P_{I}+Q_{R} P_{I_{\tau}}^{\prime}\right)\left(P_{R} Q_{R}+P_{I} Q_{I}\right) \\
\equiv & \omega^{\prime} A+B .
\end{aligned}
$$

It can be shown that

$$
\begin{aligned}
A= & -\left(P_{R}^{2}+P_{I}^{2}\right) Q_{R_{\omega}}^{\prime} Q_{I}-\left(Q_{R}^{2}+Q_{I}^{2}\right) P_{R} P_{I_{\omega}}^{\prime}+P_{R_{\omega}}^{\prime} P_{I}\left(Q_{R}^{2}+Q_{I}^{2}\right) \\
& +\left(P_{R}^{2}+P_{I}^{2}\right) Q_{R} Q_{\omega}^{\prime} \\
= & \left(P_{R_{\omega}}^{\prime} P_{I}-P_{R} P_{I_{\omega}}^{\prime}\right)|P(i \omega, \tau)|^{2}-\left(Q_{R_{\omega}}^{\prime} Q_{I}-Q_{R} Q_{I_{\omega}}^{\prime}\right)|P(i \omega, \tau)|^{2}
\end{aligned}
$$

and

$$
\begin{aligned}
B & =-\left(P_{R}^{2}+P_{I}^{2}\right)\left(Q_{R_{\tau}}^{\prime} Q_{I}-Q_{R} Q_{I_{\tau}}^{\prime}\right)+\left(Q_{R}^{2}+Q_{I}^{2}\right)\left(P_{R_{\tau}}^{\prime} P_{I}-P_{R} P_{I_{\tau}}^{\prime}\right) \\
& =|P(i \omega, \tau)|^{2}\left(Q_{R} Q_{I_{\tau}}^{\prime}-Q_{R_{\tau}}^{\prime} Q_{I}\right)-|P(i \omega, \tau)|^{2}\left(P_{R} P_{I_{\tau}}^{\prime}-P_{R_{\tau}}^{\prime} P_{I}\right) .
\end{aligned}
$$

Hence, from (2.36)-(2.38) we obtain

$$
\begin{aligned}
\theta^{\prime}(\tau)= & -\frac{\omega^{\prime}\left[\left(P_{I_{\omega}}^{\prime} P_{R}-P_{R_{\omega}}^{\prime} P_{I}\right)-\left(Q_{R} Q_{I_{\omega}}^{\prime}-Q_{R_{\omega}}^{\prime} Q_{I}\right)\right]}{|P(i \omega, \tau)|^{2}} \\
& -\frac{\left[\left(P_{R} P_{I_{\tau}}^{\prime}-P_{R_{\tau}}^{\prime} P_{I}\right)-\left(Q_{R} Q_{I_{\tau}}^{\prime}-Q_{R_{\tau}}^{\prime} Q_{I}\right)\right]}{|P(i \omega, \tau)|^{2}}=-\frac{U \omega^{\prime}+V}{|P(i \omega, \tau)|^{2}} .
\end{aligned}
$$


Therefore, if we substitute (2.39) evaluated at $\tau^{*}$ in (2.32), and we compare the result with (2.31), we find that

$$
\operatorname{sign}\left\{\left.\frac{d \operatorname{Re} \lambda}{d \tau}\right|_{\lambda=i \omega\left(\tau^{*}\right)}\right\}=\operatorname{sign}\left\{F_{\omega}^{\prime}\left(\omega, \tau^{*}\right)\right\} \operatorname{sign}\left\{S_{n}^{\prime}\left(\tau^{*}\right)\right\},
$$

thus completing the proof.

Remark 2.1. Assume that $\theta(\tau) \in(0,2 \pi), \tau \in I$, where $\theta(\tau)$ is defined by $(2.16)$. According to $(2.35)$, we can rewrite $(2.16)$ as

$$
\sin \theta(\tau)=\frac{\psi(\tau)}{|Q(i \omega, \tau)|^{2}}, \quad \cos \theta(\tau)=-\frac{\varphi(\tau)}{|Q(i \omega, \tau)|^{2}}, \quad \tau \in I,
$$

where $\psi, \varphi$ are continuous and differentiable functions of $\tau$ such that $\psi^{2}+\varphi^{2}=$ $|P(i \omega, \tau)|^{4}$ and $|Q(i \omega, \tau)|^{2}=|P(i \omega, \tau)|^{2}$ for $\tau \in I$. Hence, we have

$$
\begin{aligned}
& \theta(\tau)=\arctan (-\psi(\tau) / \varphi(\tau)) \quad \text { if } \quad \sin \theta(\tau)>0, \cos \theta(\tau)>0 \\
& \theta(\tau)=\pi / 2 \quad \text { if } \quad \sin \theta(\tau)=1, \cos \theta(\tau)=0 \\
& \theta(\tau)=\pi+\arctan (-\psi(\tau) / \varphi(\tau)) \quad \text { if } \quad \cos \theta(\tau)<0 \\
& \theta(\tau)=3 \pi / 2 \quad \text { if } \quad \sin \theta(\tau)=-1, \cos \theta(\tau)=0 \\
& \theta(\tau)=2 \pi+\arctan (-\psi(\tau) / \varphi(\tau)) \quad \text { if } \quad \sin \theta(\tau)<0, \cos \theta(\tau)>0
\end{aligned}
$$

It is easy to see that the function $\theta(\tau)$ defined above is continuous on $I$. Furthermore $\theta^{\prime}(\tau)$ is well defined for $\theta(\tau) \in(0,2 \pi)$ and it is indeed given by (2.34). Observe that if $\theta(\tau) \neq \pi / 2,3 \pi / 2$, then $\varphi(\tau) \neq 0$, and (2.34) simply follows from (2.41). When $\theta(\tau)=\pi / 2,3 \pi / 2$, we have $\varphi(\tau)=0$. In this case, we compute $\theta^{\prime}(\tau)$ directly from (2.40) and obtain

$$
-(\sin (\theta(\tau))) \theta^{\prime}(\tau)=\left(-\varphi(\tau) /\left(\psi^{2}(\tau)+\varphi^{2}(\tau)\right)^{1 / 2}\right)^{\prime} .
$$

Since $\sin \theta(\tau) \neq 0$ (i.e., $\psi(\tau) \neq 0$ ), it is easy to see that (2.42) implies (2.34) as well.

Therefore, if $\theta(\tau) \in(0,2 \pi), \tau \in I$, then $\theta(\tau)$ is continuous and differentiable. If in addition, $\omega(\tau)$ is positive, continuous, and differentiable on $I$, then functions $\tau_{n}(\tau)$ and $S_{n}(\tau), n \in \mathbf{N}_{0}$, are all continuous and differentiable.

Remark 2.2. Instead of looking for zeros of $S_{n}$, we can look for the zeros of, say, $Z_{n}=\omega S_{n}=\omega \tau-\theta(\tau)-2 n \pi=Z_{0}-2 n \pi$. Since $\omega>0$, they have the same zeros, and all the functions $Z_{n}$ have the same shape as $Z_{0}$ (they are simply shifted down by $2 n \pi)$. Furthermore it is easy to check that $\operatorname{sign}\left(S_{n}^{\prime}\right)=\operatorname{sign}\left(Z_{n}^{\prime}\right)$ when considering the derivative with respect to $\tau$ at the same zero as $S_{n}$ and $Z_{n}$. In most cases of applications, we can assume that $I=\left[0, \tau_{1}\right)$ with $\omega(0)>0$ and $\omega(\tau) \rightarrow 0$ as $\tau \rightarrow \tau_{1}$. Then clearly $Z_{n}(0)<0$ and $Z_{n}(\tau)<0$ as $\tau \rightarrow \tau_{1}$. Hence either $Z_{n}$ is negative or it has an even number of zeros (taking into account multiplicity of the zeros).

3. First order characteristic equation. In this section, we consider the first order characteristic equation

$$
D(\lambda, \tau)=0,
$$

where

$$
D(\lambda, \tau)=a(\tau) \lambda+b(\tau)+c(\tau) e^{-\lambda \tau},
$$


which belongs to the general class

$$
D(\lambda, \tau)=P_{n}(\lambda, \tau)+Q_{m}(\lambda, \tau) e^{-\lambda \tau},
$$

where $P_{n}, Q_{m}$ are polynomials in $\lambda$ with $n>m$. In our case $P(\lambda, \tau):=P_{n}(\lambda, \tau)=$ $a(\tau) \lambda+b(\tau)$ is a first order polynomial in $\lambda, Q(\lambda, \tau):=Q_{m}(\lambda, \tau)=c(\tau)$. The coefficients $a, b, c$ are real smooth functions of $\tau$ assumed to have continuous derivatives in $\tau$ and

$$
b(\tau)+c(\tau) \neq 0 \quad \forall \tau \geq 0 .
$$

Due to assumption (3.4), $\lambda=0$ cannot be a root of (3.1) and a stability switch (or a cross of the imaginary axis) necessarily occurs with $\lambda= \pm i \omega$ with $\omega>0$. Without loss of generality assume $\lambda=i \omega, \omega>0$, as a root of (3.2). Hence at $\lambda=i \omega$ we have

$$
P(i \omega, \tau)=b(\tau)+i \omega a(\tau), \quad Q(i \omega, \tau)=c(\tau),
$$

i.e.,

$$
F(\omega, \tau)=|P(i \omega, \tau)|^{2}-|Q(i \omega, \tau)|^{2}=\omega^{2} a^{2}+b^{2}-c^{2},
$$

from which $F(\omega, \tau)=0$ gives a solution for $\omega(\tau)>0$ :

$$
\omega(\tau)=\left(\frac{c^{2}(\tau)-b^{2}(\tau)}{a^{2}(\tau)}\right)^{1 / 2},
$$

which is defined if $|c(\tau)|>|b(\tau)|$ and $a(\tau) \neq 0$. Furthermore, since $P_{R}(i \omega, \tau)=b(\tau)$, $P_{I}(i \omega, \tau)=\omega a(\tau), Q_{R}(i \omega, \tau)=c(\tau), Q_{I}(i \omega, \tau)=0,(2.16)$ give

$$
\sin \theta(\tau)=\frac{\omega(\tau) a(\tau)}{c(\tau)}, \quad \cos \theta(\tau)=-\frac{b(\tau)}{c(\tau)} .
$$

Let

$$
I=\{\tau: \tau \geq 0, a(\tau) \neq 0 \quad \text { and } \quad|b(\tau)|<|c(\tau)|\} .
$$

Let $\theta(\tau) \in I$ be the solution of (3.8). Then a stability switch may occur, through the roots $\lambda= \pm i \omega(\tau)$, where $\omega(\tau)>0$ is given by (3.7), at the $\tau$ values

$$
\tau_{n}=\frac{\theta(\tau)+n 2 \pi}{\omega(\tau)}
$$

for $n \in \mathbf{N}_{0}:=\{0,1,2, \ldots\}$. Then for each $n \in \mathbf{N}_{0}$ (3.9) defines the maps $\tau_{n}: I \rightarrow \mathbf{R}_{+0}$, and the stability switch may occur only for the $\tau$ values at which

$$
\tau_{n}(\tau)=\tau \quad \text { for some } \quad n \in \mathbf{N}_{0} .
$$

Hence (3.7), (3.8) define the maps (3.9), and the occurrence of stability switches takes place at the zeros of the functions

$$
S_{n}(\tau):=\tau-\tau_{n}(\tau), \quad n \in \mathbf{N}_{0} .
$$

Remark 3.1. We remark here that for $\tau \in I, \theta(\tau)$ is continuous and differentiable in $\tau$. To see this, we observe that due to (3.4), we have $\cos \theta(\tau) \neq 1$ for $\tau \in I$. Hence 
for $\tau \in I, \theta(\tau) \neq 0,2 \pi$. This shows that $\theta(\tau)$ is continuous and differentiable. As a result, we see that $\tau_{n}(\tau)$ are also continuous and differentiable for $\tau \in I$. Now we want to see if it is possible to determine the direction in which the pair of imaginary roots $\lambda= \pm i \omega\left(\tau^{*}\right)$ (where $\tau^{*}$ is such that $S_{n}\left(\tau^{*}\right)=0$ for some $n$ ) crosses the imaginary axis as $\tau$ increases. In view of the fact that it is now quite straightforward to generate the graphs of $S_{n}(\tau)$ by popular software such as Maple, we want to connect the rather abstract value of

$$
R(\tau):=\operatorname{sign}\left\{\left.\frac{d \operatorname{Re} \lambda}{d \tau}\right|_{\lambda=i \omega\left(\tau^{*}\right)}\right\}
$$

with the intuitive and easy to use one

$$
S(\tau):=\operatorname{sign}\left\{\left.\frac{d S_{n}(\tau)}{d \tau}\right|_{\tau=\tau^{*}}\right\}=\operatorname{sign}\left\{1-\left.\frac{d \tau_{n}(\tau)}{d \tau}\right|_{\tau=\tau^{*}}\right\} .
$$

Observe that

$$
F_{\omega}^{\prime}(\omega, \tau)=2 \omega a^{2}>0
$$

since $\omega(\tau)>0$. Therefore, (2.21) in Theorem 2.2 becomes

$$
\operatorname{sign}\left\{\left.\frac{d \operatorname{Re} \lambda}{d \tau}\right|_{\lambda=i \omega\left(\tau^{*}\right)}\right\}=\operatorname{sign}\left\{\left.\frac{d S_{n}(\tau)}{d \tau}\right|_{\tau=\tau^{*}}\right\} .
$$

Hence, we have the following.

THEOREM 3.1. The characteristic equation (3.1) admits a pair of simple and conjugate roots $\lambda_{+}\left(\tau^{*}\right)=i \omega\left(\tau^{*}\right), \lambda_{-}\left(\tau^{*}\right)=-i \omega\left(\tau^{*}\right), \omega\left(\tau^{*}\right)>0$, at $\tau^{*} \in I$ if $S_{n}\left(\tau^{*}\right)=$ 0 , for some $n \in \mathbf{N}_{0}$. This pair of simple conjugate pure imaginary roots crosses the imaginary axis from left to right if $\delta\left(\tau^{*}\right)>0$ and crosses the imaginary axis from right to left if $\delta\left(\tau^{*}\right)<0$, where

$$
\delta\left(\tau^{*}\right)=\operatorname{sign}\left\{\left.\frac{d \operatorname{Re} \lambda}{d \tau}\right|_{\lambda=i \omega\left(\tau^{*}\right)}\right\}=\operatorname{sign}\left\{\left.\frac{d S_{n}(\tau)}{d \tau}\right|_{\tau=\tau^{*}}\right\} .
$$

The following analytical result on $R(\tau)$ is useful for determining analytically the $\tau$ values at which a stability switch occurs.

TheOREm 3.2. For the characteristic equation (3.1),

$$
\begin{aligned}
\operatorname{sign}\left\{\left.\frac{d \operatorname{Re} \lambda}{d \tau}\right|_{\lambda=i \omega\left(\tau^{*}\right)}\right\}= & \operatorname{sign}\left\{a^{2}(\tau) \omega(\tau) \omega^{\prime}(\tau)\left(a(\tau) b(\tau)+c(\tau)^{2} \tau\right)\right. \\
& \left.+\omega^{2}(\tau) a^{2}(\tau)\left(a^{\prime}(\tau) b(\tau)-a(\tau) b^{\prime}(\tau)+c^{2}(\tau)\right)\right\}
\end{aligned}
$$

Proof. Denote by $a^{\prime}, b^{\prime}, c^{\prime}$ the derivatives of $a(\tau), b(\tau), c(\tau)$ with respect to $\tau$. Differentiating (3.1) with respect to $\tau$ we obtain

$$
\frac{d \lambda}{d \tau}=\frac{\lambda c(\tau) e^{-\lambda \tau}-\left(a^{\prime}(\tau) \lambda+b^{\prime}(\tau)+c^{\prime}(\tau) e^{-\lambda \tau}\right)}{a(\tau)-c(\tau) \tau e^{-\lambda \tau}} .
$$

It is convenient to consider $(d \lambda / d \tau)^{-1}$. Hence, from (3.17) we have

$$
\left(\frac{d \lambda}{d \tau}\right)^{-1}=\frac{a(\tau) e^{\lambda \tau}-c(\tau) \tau}{\lambda c(\tau)-\left(a^{\prime}(\tau) \lambda+b^{\prime}(\tau)\right) e^{\lambda \tau}-c^{\prime}(\tau)},
$$


where, due to $(3.1)$

$$
e^{\lambda \tau}=-\frac{c(\tau)}{a(\tau) \lambda+b(\tau)} .
$$

Therefore, substituting (3.19) in (3.18), we have

$$
\left(\frac{d \lambda}{d \tau}\right)^{-1}=\frac{-\frac{a(\tau) c(\tau)}{a(\tau) \lambda+b(\tau)}-c(\tau) \tau}{\lambda c(\tau)+\frac{c(\tau)\left(a^{\prime}(\tau) \lambda+b^{\prime}(\tau)\right)}{a(\tau) \lambda+b(\tau)}-c^{\prime}(\tau)} .
$$

Now, we compute (3.20) at $\lambda=i \omega(\tau)$. We have

$$
\left.\left(\frac{d \lambda}{d \tau}\right)^{-1}\right|_{\lambda=i \omega(\tau)}=\frac{-\frac{a c(b-i \omega a)}{\left(\omega^{2} a^{2}+b^{2}\right)}-c \tau}{i \omega c+\frac{c\left(i \omega a^{\prime}+b^{\prime}\right)(b-i \omega a)}{\omega^{2} a^{2}+b^{2}}-c^{\prime}} .
$$

Since $\omega^{2}(\tau) a^{2}(\tau)+b^{2}(\tau)=c^{2}(\tau)$ for any $\tau \in I$, we obtain

$$
\left.\left(\frac{d \lambda}{d \tau}\right)^{-1}\right|_{\lambda=i \omega(\tau)}=\frac{-\left(a b+c^{2} \tau\right)+i \omega a^{2}}{\omega^{2} a a^{\prime}+b b^{\prime}-c c^{\prime}+i \omega\left(a^{\prime} b-a b^{\prime}+c^{2}\right)} .
$$

Since $F(\omega, \tau)=0$ for all $\tau \in I$, we obtain

$$
-\omega \omega^{\prime} a^{2}=\omega^{2} a a^{\prime}+b b^{\prime}-c c^{\prime}
$$

for all $\tau \in I$, which substituted in (3.22) provides

$$
\left.\left(\frac{d \lambda}{d \tau}\right)^{-1}\right|_{\lambda=i \omega(\tau)}=\frac{-\left(a(\tau) b(\tau)+c(\tau)^{2} \tau\right)+i \omega(\tau) a(\tau)^{2}}{-a^{2}(\tau) \omega(\tau) \omega^{\prime}(\tau)+i \omega(\tau)\left(a^{\prime}(\tau) b(\tau)-a(\tau) b^{\prime}(\tau)+c^{2}(\tau)\right)}
$$

Therefore, we have

$$
\begin{aligned}
R(\tau)=\operatorname{sign} & \left\{a^{2}(\tau) \omega(\tau) \omega^{\prime}(\tau)\left(a(\tau) b(\tau)+c(\tau)^{2} \tau\right)+\omega^{2}(\tau) a^{2}(\tau)\left(a^{\prime}(\tau) b(\tau)\right.\right. \\
& \left.\left.-a(\tau) b^{\prime}(\tau)+c^{2}(\tau)\right)\right\},
\end{aligned}
$$

proving the theorem.

Example. As an example of first order characteristic equations with delay dependent coefficients, we consider the first model with time delay (simpler one) introduced by Bence and Nisbet [3] for a population of sessile invertebrates. (This population was previously studied by Roughgarden, Iwasa, and Baxter [25] and Roughgarden and Iwasa [24] in terms of different mathematical models.) This model is a two-stage model in which the population is divided into an adult population, which is explicitly modeled, and a juvenile population which is modeled implicitly. The model takes the form

$$
\frac{d A}{d t}=s e^{-m_{J} \tau} \max \left\{0,1-a_{A} A(t-\tau)\right\}-m_{A} A(t),
$$

where $A(t)$ represents the adult population, $s$ is the settlement rate of juveniles, $e^{-m_{J} \tau}$ is the through-stage survival probability of juveniles, $m_{A}$ is the mortality rate of adults, and $\tau$ is the fixed time delay between settlement and recruitment into 
the adult population. Finally $a_{A}>0$ is the amount of space occupied by an adult individual. The characteristic equation at steady state takes the form (3.1) with

$$
a(\tau)=1, \quad b(\tau)=m_{A}, \quad c(\tau)=a_{A} s e^{-m_{J} \tau},
$$

and $\tau \in \mathbf{R}_{+0}$. Furthermore,

$$
b(0)+c(0)=m_{A}+a_{A} s>0,
$$

thus ensuring that at $\tau=0$ we have one negative eigenvalue.

Let $\omega(\tau)$ be the positive solution of

$$
\omega^{2}(\tau)=a_{A}^{2} s^{2} e^{-2 m_{J} \tau}-m_{A}^{2}
$$

which exists provided that

$$
a_{A} s>m_{A}, \quad \tau<\tau_{1}:=\frac{1}{m_{J}} \log \left(\frac{a_{A} s}{m_{A}}\right) .
$$

Then, eigenvalues $\lambda_{+}=i \omega(\tau), \lambda_{-}=-i \omega(\tau), \omega(\tau)>0$, can only occur for delays $\tau$ in the interval $I=\left(0, \tau_{1}\right)$. No stability switches for $\tau \geq \tau_{1}$. It is interesting to determine the values of $\tau$ at which $R(\tau)=1$ and those at which $R(\tau)=-1$. To this end we can use formula (3.16)

$$
R(\tau)=\operatorname{sign}\left\{a^{2} \omega \omega^{\prime}\left(a b+c^{2} \tau\right)+\omega^{2} a 2\left(a^{\prime} b-a b^{\prime}\right)+\omega^{2} c^{2}\right\},
$$

where $a, b, c$ are coefficients in (3.27) and $\omega$ is defined by (3.28). From (3.27) we see that $a^{\prime}=b^{\prime}=0, a b=m_{A}$,

$$
\omega \omega^{\prime}=-m_{J} a_{A}^{2} s^{2} e^{-2 m_{J} \tau}=-m_{J} c^{2} .
$$

Substituting (3.30) in (3.16) we obtain

$$
\begin{aligned}
R(\tau) & =\operatorname{sign}\left\{-m_{J} c^{2}\left(m_{A}+c^{2} \tau\right)+\omega^{2} c^{2}\right\} \\
& =\operatorname{sign}\left\{-m_{A}\left(m_{J}+m_{A}\right)-c^{2}\left(m_{J} \tau-1\right)\right\} .
\end{aligned}
$$

Then if $\left(1 / m_{J}\right)<\tau_{1},(3.31)$ shows that in the interval $\left(\left(1 / m_{J}\right), \tau_{1}\right)$ we have $R(\tau)=$ -1 , i.e., possible stability switches can only occur toward stability. Assume that $\tau<\min \left\{\left(1 / m_{J}\right), \tau_{1}\right\}$. Then (3.31) takes the form

$$
R(\tau)=\operatorname{sign}\left\{\left(1-m_{J} \tau\right) a_{A}^{2} s^{2} e^{-2 m_{J} \tau}-m_{A}\left(m_{J}+m_{A}\right)\right\},
$$

which yields the following conclusions:

(i) If the parameters satisfy

$$
m_{A}<a_{A} s<m_{A}\left(1+\frac{m_{J}}{m_{A}}\right)^{1 / 2},
$$

then for all $\tau \in\left[0, \tau_{1}\right)$ we have $R(\tau)=-1$. In such cases, a stability switch from unstable to stable may occur. Since at $\tau=0$ the steady state is asymptotically stable, then it remains asymptotically stable for all $\tau \in\left[0, \tau_{1}\right)$.

(ii) Assume that the parameters satisfy

$$
a_{A} s>m_{A}\left(1+\frac{m_{J}}{m_{A}}\right)^{1 / 2} .
$$




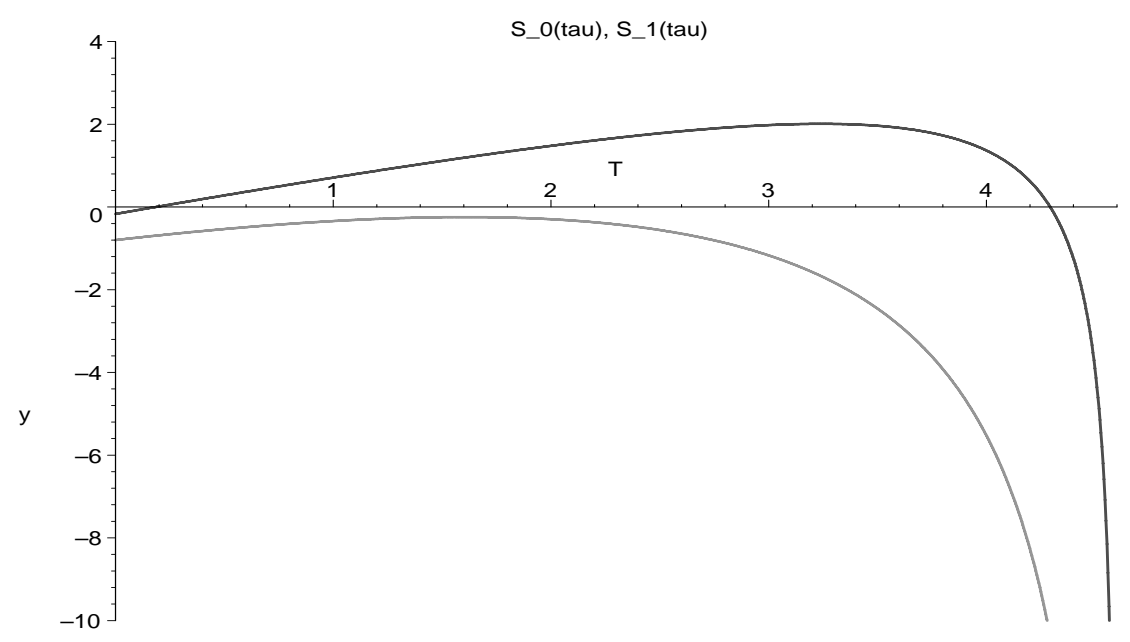

FIG. 1. Graph of stability switch in terms of time delay for the first model of Bence and Nisbet [3]. The top curve is $S_{0}(\tau)$.

Then there exists a $\tau_{c}, 0<\tau_{c}<\tau_{1}$, such that

$$
\operatorname{sign}\left\{\left.\frac{d \operatorname{Re} \lambda}{d \tau}\right|_{\lambda=i \omega(\tau)}\right\}>0
$$

in $\left(0, \tau_{c}\right)$, vanishes at $\tau_{c}$, and is negative in $\left(\tau_{c}, \tau_{1}\right) \cdot \tau_{c}$ is the unique zero of

$$
\varphi(\tau):=\left(1-m_{J} \tau\right) a_{A}^{2} s^{2} e^{-2 m_{J} \tau}-m_{A}\left(m_{J}+m_{A}\right), \quad \tau \in\left(0, \tau_{1}\right) .
$$

The statements of (i), (ii) are helpful in choosing the parameters to perform relevant numerical simulations. In Figure 1, we plot the graph of the map $S_{0}(\tau)$ versus $\tau$ in the interval $I=\left[0, \tau_{1}\right)$ for the following set of parameters satisfying (3.34):

$$
a_{A}=1, \quad m_{A}=1, \quad m_{J}=0.5, \quad s=10,
$$

with $\tau_{1}=4.605$. The graph of $S_{0}(\tau)$ versus $\tau$ in Figure 1 shows that $S_{0}(\tau)$ has two zeros, the first at the value $\tau_{01}=0.20$, the second at the value $\tau_{02}=4.24$, and $S_{1}(\tau)<0$ on $\left(0, \tau_{1}\right)$. According to Theorem 3.1 at $\tau_{01}$ a stability switch occurs toward instability whereas at $\tau_{02}$ the stability switch occurs toward stability. Hence, for the model by Bence and Nisbet [3], as confirmed by other computer simulations, intermediate delays $(\tau \in(0.2,4.24))$ show a destabilizing effect on the steady state, whereas large delays $(\tau>4.24)$ have a stabilizing one. For $\tau \in\left(\tau_{01}, \tau_{02}\right)$, the steady state is unstable whereas it is asymptotically stable for $0 \leq \tau<\tau_{01}$ and for any $\tau>\tau_{02}$. These results are in agreement with our computer simulations using XPP.

4. Second order characteristic equation. In this section, we consider the characteristic equation

$$
D(\lambda, \tau):=\lambda^{2}+a(\tau) \lambda+b(\tau) \lambda e^{-\lambda \tau}+c(\tau)+d(\tau) e^{-\lambda \tau}=0
$$


$\tau \in \mathbf{R}_{+0}$ and $a(\tau), b(\tau), c(\tau), d(\tau): \mathbf{R}_{+0} \rightarrow \mathbf{R}$ are differentiable functions of class $C^{1}\left(\mathbf{R}_{+0}\right)$ such that $c(\tau)+d(\tau) \neq 0$ for all $\tau \in \mathbf{R}_{+0}$, and for any $\tau, b(\tau), d(\tau)$ are not simultaneously zero. We have

$$
P(\lambda, \tau):=P_{n}(\lambda, \tau)=\lambda^{2}+a(\tau) \lambda+c(\tau), \quad Q(\lambda, \tau):=Q_{m}(\lambda, \tau)=b(\tau) \lambda+d(\tau) .
$$

We assume that $P_{n}(\lambda, \tau)$ and $Q_{m}(\lambda, \tau)$ cannot have common imaginary roots. That is, for any real number $\omega$,

$$
P_{n}(i \omega, \tau)+Q_{m}(i \omega, \tau) \neq 0
$$

We have

$$
F(\omega, \tau)=|P(i \omega, \tau)|^{2}-|Q(i \omega, \tau)|^{2}=\left(c-\omega^{2}\right)^{2}+\omega^{2} a^{2}-\left(\omega^{2} b^{2}+d^{2}\right) .
$$

Hence, $F(\omega, \tau)=0$ implies

$$
\omega^{4}-\omega^{2}\left(b^{2}+2 c-a^{2}\right)+\left(c^{2}-d^{2}\right)=0,
$$

and its roots are given by

$$
\omega_{+}^{2}=\frac{1}{2}\left\{\left(b^{2}+2 c-a^{2}\right)+\Delta^{1 / 2}\right\}, \quad \omega_{-}^{2}=\frac{1}{2}\left\{\left(b^{2}+2 c-a^{2}\right)-\Delta^{1 / 2}\right\},
$$

where

$$
\Delta=\left(b^{2}+2 c-a^{2}\right)^{2}-4\left(c^{2}-d^{2}\right) .
$$

Therefore, the following holds:

$$
2 \omega_{ \pm}^{2}-\left(b^{2}+2 c-a^{2}\right)= \pm \Delta^{1 / 2} .
$$

Furthermore, $P_{R}(i \omega, \tau)=c(\tau)-\omega^{2}(\tau), P_{I}(i \omega, \tau)=\omega(\tau) a(\tau), Q_{R}(i \omega, \tau)=d(\tau)$, $Q_{I}(i \omega, \tau)=\omega(\tau) b(\tau)$. Hence $(2.16)$ becomes

$$
\sin \theta(\tau)=\frac{-\left(c-\omega^{2}\right) \omega b+\omega a d}{\omega^{2} b^{2}+d^{2}}, \quad \cos \theta(\tau)=-\frac{\left(c-\omega^{2}\right) d+\omega^{2} a b}{\omega^{2} b^{2}+d^{2}},
$$

which jointly with (4.4) defines the maps (2.19). Now, from (4.3) we have

$$
\begin{aligned}
F_{\omega}^{\prime}(\omega, \tau) & =2\left(c-\omega^{2}\right)(-2 \omega)+2 \omega a^{2}-2 \omega b^{2} \\
& =2 \omega\left[2 \omega^{2}-\left(b^{2}+2 c-a^{2}\right)\right] \\
& =2 \omega_{ \pm}\left[ \pm \Delta^{1 / 2}\right]
\end{aligned}
$$

where $\omega_{ \pm}(\tau)>0$. Hence (2.21) in Theorem 2.2 becomes

$$
\operatorname{sign}\left\{\left.\frac{d \operatorname{Re} \lambda}{d \tau}\right|_{\lambda=i \omega_{ \pm}}\right\}=\operatorname{sign}\left\{ \pm \Delta^{1 / 2}\right\} \operatorname{sign}\left\{\left.\frac{d S_{n}(\tau)}{d \tau}\right|_{\tau=\tau^{*}}\right\} .
$$

This proves the following theorem.

THEOREM 4.1. The characteristic equation (4.1) has a pair of simple and conjugate pure imaginary roots $\lambda= \pm i \omega\left(\tau^{*}\right), \omega\left(\tau^{*}\right)$ real, at $\tau^{*} \in I$ if $S_{n}\left(\tau^{*}\right)=\tau^{*}-\tau_{n}\left(\tau^{*}\right)=$ 0 for some $n \in \mathbf{N}_{0}$. If $\omega\left(\tau^{*}\right)=\omega_{+}\left(\tau^{*}\right)$, this pair of simple conjugate pure imaginary 
roots crosses the imaginary axis from left to right if $\delta_{+}\left(\tau^{*}\right)>0$ and crosses the imaginary axis from right to left if $\delta_{+}\left(\tau^{*}\right)<0$, where

$$
\delta_{+}\left(\tau^{*}\right):=\operatorname{sign}\left\{\left.\frac{d \operatorname{Re} \lambda}{d \tau}\right|_{\lambda=i \omega_{+}\left(\tau^{*}\right)}\right\}=\operatorname{sign}\left\{\left.\frac{d S_{n}(\tau)}{d \tau}\right|_{\tau=\tau^{*}}\right\} .
$$

If $\omega\left(\tau^{*}\right)=\omega_{-}\left(\tau^{*}\right)$, this pair of simple conjugate pure imaginary roots crosses the imaginary axis from left to right if $\delta_{-}\left(\tau^{*}\right)>0$ and crosses the imaginary axis from right to left if $\delta_{-}\left(\tau^{*}\right)<0$, where

$$
\delta_{-}\left(\tau^{*}\right):=\operatorname{sign}\left\{\left.\frac{d \operatorname{Re} \lambda}{d \tau}\right|_{\lambda=i \omega_{-}\left(\tau^{*}\right)}\right\}=-\operatorname{sign}\left\{\left.\frac{d S_{n}(\tau)}{d \tau}\right|_{\tau=\tau^{*}}\right\} .
$$

We remark that if $\omega_{+}\left(\tau^{*}\right)=\omega_{-}\left(\tau^{*}\right)=\omega\left(\tau^{*}\right)$, then $\Delta\left(\tau^{*}\right)=0$ and

$$
\operatorname{sign}\left\{\left.\frac{d \operatorname{Re} \lambda}{d \tau}\right|_{\lambda=i \omega\left(\tau^{*}\right)}\right\}=0 .
$$

The same is true when $S_{n}^{\prime}\left(\tau^{*}\right)=0$. The following result can be useful in identifying values of $\tau$ where stability switches may take place. (In using this theorem, the plus or minus signs are to be used consistently on both sides of the equations.)

TheOREM 4.2. Assume that for all $\tau \in I, \omega(\tau)$ is defined as a solution of (4.4). Then

$$
\delta_{ \pm}(\tau)=\operatorname{sign}\left\{ \pm \Delta^{1 / 2}(\tau)\right\} \operatorname{sign} D_{ \pm}(\tau)
$$

where

$$
\begin{aligned}
D_{ \pm}(\tau)= & \omega_{ \pm}^{2}\left[\left(\omega_{ \pm}^{2} b^{2}+d^{2}\right)+a^{\prime}\left(c-\omega_{ \pm}^{2}\right)+b d^{\prime}-b^{\prime} d-a c^{\prime}\right] \\
& +\omega_{ \pm} \omega_{ \pm}^{\prime}\left[\tau\left(\omega_{ \pm}^{2} b^{2}+d^{2}\right)-b d+a\left(c-\omega_{ \pm}^{2}\right)+2 \omega_{ \pm}^{2} a\right]
\end{aligned}
$$

for all $\tau \in I$.

Proof. Let us differentiate with respect to $\tau$ the characteristic equation (4.1). We obtain

$$
\left(\frac{d \lambda}{d \tau}\right)^{-1}=\frac{-\frac{2 \lambda+a}{\lambda^{2}+a \lambda+c}+\frac{b}{b \lambda+d}-\tau}{\lambda+\frac{a^{\prime} \lambda+c^{\prime}}{\lambda^{2}+a \lambda+c}-\frac{b^{\prime} \lambda+d^{\prime}}{b \lambda+d}}
$$

Now, let $\lambda=i \omega(\tau)$ where $\omega(\tau)>0$ satisfies (4.4). Hence, we have

$$
\begin{aligned}
\left.\operatorname{Re}\left(\frac{d \lambda}{d \tau}\right)^{-1}\right|_{\lambda=i \omega} & =\operatorname{Re}\left\{\frac{-\frac{(2 i \omega+a)\left(\left(c-\omega^{2}\right)-i \omega a\right)}{\left(c-\omega^{2}\right)^{2}+\omega^{2} a^{2}}+\frac{b(d-i \omega b)}{d^{2}+\omega^{2} b^{2}}-\tau}{i \omega+\frac{\left(i \omega a^{\prime}+c^{\prime}\right)\left(\left(c-\omega^{2}\right)-i \omega a\right)}{\left(c-\omega^{2}\right)^{2}+\omega^{2} a^{2}}-\frac{\left(i \omega b^{\prime}+d^{\prime}\right)(d-i \omega b)}{d^{2}+\omega^{2} b^{2}}}\right\} \\
& =\operatorname{Re}\left\{\frac{\left[b d-\tau\left(d^{2}+\omega^{2} b^{2}\right)-2 \omega^{2} a-a\left(c-\omega^{2}\right)\right]+i\left[\omega\left(2 \omega^{2}-\left(b^{2}+2 c-a^{2}\right)\right)\right]}{\left[\omega^{2} a a^{\prime}+c^{\prime}\left(c-\omega^{2}\right)-\omega^{2} b b^{\prime}-d d^{\prime}\right]+i\left[\omega\left(\left(d^{2}+\omega^{2} b^{2}\right)+a^{\prime}\left(c-\omega^{2}\right)-a c^{\prime}+b d^{\prime}-b^{\prime} d\right)\right]}\right\} .
\end{aligned}
$$

Therefore, from (4.16) we have

$$
\begin{aligned}
\operatorname{sign}\left\{\left.\operatorname{Re}\left(\frac{d \lambda}{d \tau}\right)^{-1}\right|_{\lambda=i \omega}\right\}=\operatorname{sign}\{[ & \left.\left(d^{2}+\omega^{2} b^{2}\right)-b d+a\left(c-\omega^{2}\right)+2 \omega^{2} a\right] \\
\times & {\left[-\omega^{2} a a^{\prime}-c^{\prime}\left(c-\omega^{2}\right)+\omega^{2} b b^{\prime}+d d^{\prime}\right] } \\
& +\omega^{2}\left[2 \omega^{2}-\left(b^{2}+2 c-a^{2}\right)\right] \\
\times & {\left.\left[\left(\omega^{2} b^{2}+d^{2}\right)+a^{\prime}\left(c-\omega^{2}\right)+b d^{\prime}-b^{\prime} d-a c^{\prime}\right]\right\} . }
\end{aligned}
$$


Differentiating both sides of (4.4) with respect to $\tau$ we obtain

$$
\omega \omega^{\prime}\left[2 \omega^{2}-\left(b^{2}+2 c-a^{2}\right)\right]=-\omega^{2} a a^{\prime}-c^{\prime}\left(c-\omega^{2}\right)+\omega^{2} b b^{\prime}+d d^{\prime} .
$$

Furthermore, $\omega(\tau)$ must be one of the two roots $\omega_{ \pm}(\tau)$ given by (4.5); therefore,

$$
2 \omega_{ \pm}^{2}-\left(b^{2}+2 c-a^{2}\right)= \pm \Delta^{1 / 2} .
$$

Combining (4.18) and (4.19) in (4.17), we obtain

$$
\begin{aligned}
& \quad \operatorname{sign}\left\{\left.\operatorname{Re}\left(\frac{d \lambda}{d \tau}\right)^{-1}\right|_{\lambda=i \omega_{ \pm}(\tau)}\right\} \\
& =\operatorname{sign}\left\{ \pm \Delta^{1 / 2}(\tau)\right\} \\
& \quad \times \operatorname{sign}\left\{\omega_{ \pm}^{2}\left[\left(\omega_{ \pm}^{2} b^{2}+d^{2}\right)+a^{\prime}\left(c-\omega_{ \pm}^{2}\right)+b d^{\prime}-b^{\prime} d-a c^{\prime}\right]\right. \\
& \left.\quad+\omega_{ \pm} \omega_{ \pm}^{\prime}\left[\tau\left(\omega_{ \pm}^{2} b^{2}+d^{2}\right)-b d+a\left(c-\omega_{ \pm}^{2}\right)+2 \omega_{ \pm}^{2} a\right]\right\} .
\end{aligned}
$$

Since

$$
\operatorname{sign}\left\{\frac{d \operatorname{Re} \lambda}{d \tau}\right\}=\operatorname{sign}\left\{\operatorname{Re}\left(\frac{d \lambda}{d \tau}\right)^{-1}\right\}
$$

the proof is completed.

Remark 4.1. In almost all the application problems that we have encountered so far that have characteristic equations of the form (4.1), often only $\omega_{+}$is feasible. In these cases, stability switches occur only at the roots of $S_{0}^{+}(\tau)=\tau-\tau_{0}^{+}(\tau)=0$, where

$$
\tau_{0}^{+}(\tau)=\theta_{+}(\tau) / \omega_{+}(\tau), \quad \tau \in I,
$$

and $\theta_{+}(\tau)$ is the solution of (4.8) when $\omega=\omega_{+}$.

However, if both $\omega_{+}$and $\omega_{-}$are feasible for $\tau \in I$, then we have the following two sequences of functions on $I$ :

$$
S_{n}^{+}(\tau)=\tau-\left(\theta_{+}(\tau)+2 n \pi\right) / \omega_{+}(\tau), \quad S_{n}^{-}(\tau)=\tau-\left(\theta_{-}(\tau)+2 n \pi\right) / \omega_{-}(\tau),
$$

where the notations are self-evident. Clearly $S_{n}^{+}(\tau)>S_{n+1}^{+}(\tau)$ and $S_{n}^{-}(\tau)>S_{n+1}^{-}(\tau)$ for all $n \in \mathbf{N}_{0}, \tau \in I$. In addition to this, we have the following simple statement.

Theorem 4.3. Assume that $S_{0}^{+}(\tau)>S_{0}^{-}(\tau)$ on $I$. Then $S_{n}^{+}(\tau)>S_{n}^{-}(\tau)$ on $I$ for all $n \in \mathbf{N}_{0}$.

Proof. It is easy to see that $S_{0}^{+}(\tau)>S_{0}^{-}(\tau)$ on $I$ implies that

$$
\theta_{+}(\tau) / \omega_{+}(\tau)<\theta_{-}(\tau) / \omega_{-}(\tau), \quad \tau \in I .
$$

Note that $\omega_{+}(\tau)>\omega_{-}(\tau)$ on $I$; the theorem follows from (4.21).

Remark 4.2. When both $\omega_{+}$and $\omega_{-}$are feasible for $\tau \in I$, it is easy to imagine that the stability switches may depend on all real roots of $S_{n}^{+}(\tau)=0$ and $S_{n}^{-}(\tau)=0$. In such situations, one must examine all these possible real roots in order to determine the stability of the equilibrium. To illustrate this, let us consider such a scenario. Assume that Theorem 4.3 holds true and the equilibrium is asymptotically stable when $\tau=0$. Assume further that $S_{0}^{+}(\tau)=0$ at $t_{1}=\tau_{01}^{+}$and $t_{4}=\tau_{02}^{+}, S_{0}^{-}(\tau)=0$ at $t_{2}=\tau_{01}^{-}$ and $t_{3}=\tau_{02}^{-}$, and no real roots for $S_{n}^{+}(\tau)=0$ and $S_{n}^{-}(\tau)=0$ when $n>0$. Then it is easy to see (Figure 2) that $t_{1}<t_{2}<t_{3}<t_{4}$. Careful but simple examination shows 


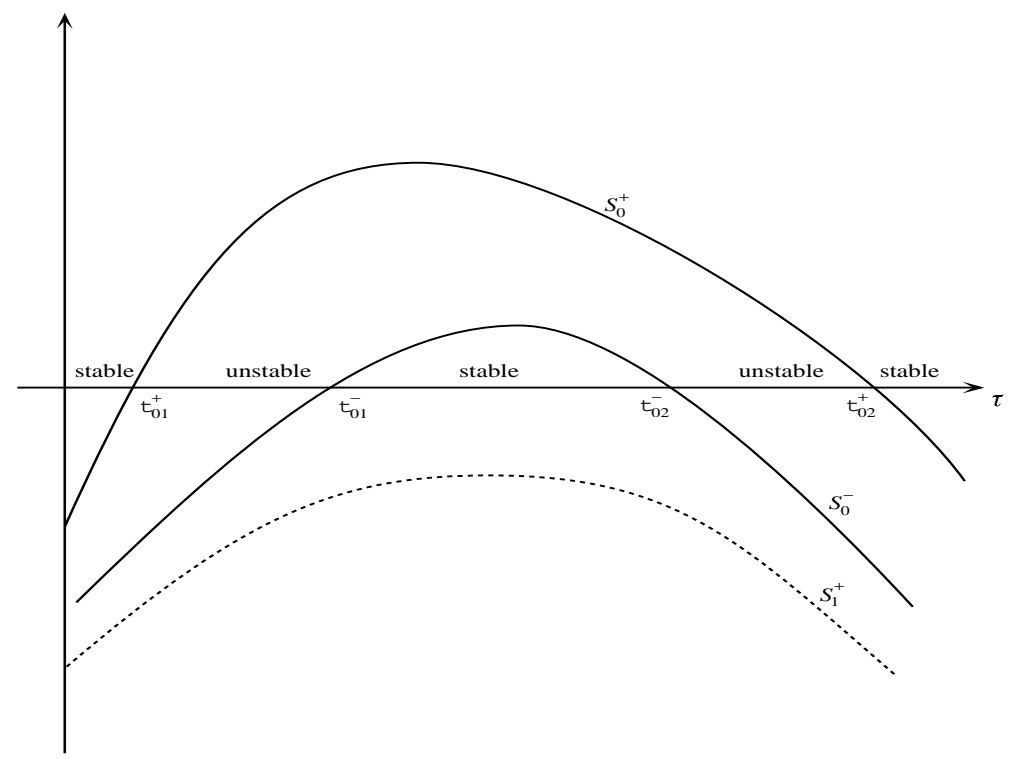

FIG. 2. Illustration for Remark 4.2.

that the equilibrium is asymptotically stable for $\tau \in\left[0, t_{1}\right) \cup\left(t_{2}, t_{3}\right) \cup\left(\left(t_{4}, \infty\right) \cap I\right)$ and is unstable for $\tau \in\left(t_{1}, t_{2}\right) \cup\left(t_{3}, t_{4}\right)$. More complicated scenarios are clearly conceivable.

Example. As an example for the second order characteristic equations with delay dependent coefficients, we consider the second model with time delay and stage structure introduced by Bence and Nisbet [3] for a population of sessile invertebrates. Again, the model is a two-stage model in which population is divided into adult and juvenile populations, both of which are explicitly modeled. The model takes the form

$$
\begin{aligned}
J^{\prime}(t) & =s\left[F(t)-e^{-m_{J} \tau} F(t-\tau)\right]-m_{J} J(t), \\
A^{\prime}(t) & =s e^{-m_{J} \tau} F(t-\tau)-m_{A} A(t), \\
F(t) & =\max \left\{0,1-a_{J} J(t)-a_{A} A(t)\right\},
\end{aligned}
$$

where $s F(t)$ represents the newly settled juveniles and $s e^{-m_{J} \tau} F(t-\tau)$ the ones that become adults. When $a_{J}=0$, this model reduces to (3.26). Here, $s, m_{g}, m_{A}, a_{A}$ are positive constants and $a_{J}$ is a nonnegative constant. This model was systematically studied by Kuang and So in [22]. It should be mentioned here that the last statement of their Theorem 4.1, which says that $S_{0}^{+}(\tau)>0$ (with respect to the positive steady state) implies that the positive steady state is unstable, is not fully justified. To be accurate, it requires the assumption that $\omega_{-}$is not feasible. The analysis and simulation (with both Maple and XPP) we conducted so far, however, indeed suggest that this assumption may in fact hold for all biologically meaningful parameters.

Let $J^{*}, A^{*}, F^{*}$ denote equilibrium population sizes of juveniles, adults, and equilibrium free space, respectively. We have

$$
J^{*}=\frac{s F^{*}}{m_{J}}\left(1-e^{-m_{J} \tau}\right), \quad A^{*}=\frac{s F^{*}}{m_{A}} e^{-m_{J} \tau} .
$$




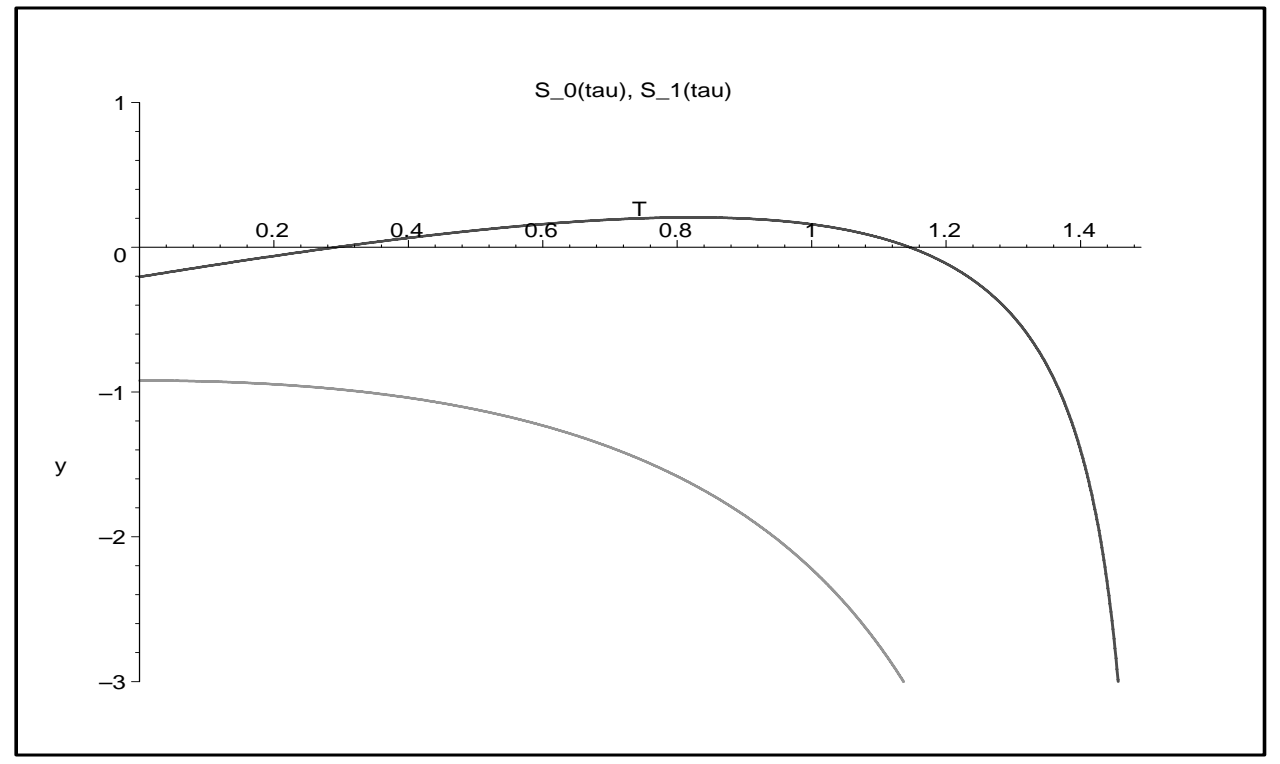

FIG. 3. Graph of stability switch in terms of time delay for the second model of Bence and Nisbet [3]. The top curve is $S_{0}(\tau)$.

It follows that $F^{*} \neq 0$ and

$$
F^{*}=1-s F^{*}\left[\frac{a_{J}}{m_{J}}\left(1-e^{-m_{J} \tau}\right)+\frac{a_{A}}{m_{A}} e^{-m_{J} \tau}\right] .
$$

Following the notation of Bence and Nisbet [3], $\sigma_{J}=s a_{J} / m_{J}, \sigma_{A}=s a_{A} / m_{A}$, we have

$$
F^{*}=\left[1+\sigma_{J}+\left(\sigma_{A}-\sigma_{J}\right) e^{-m_{J} \tau}\right]^{-1} .
$$

Thus, system (4.23)-(4.25) has a unique positive steady state $\left(J^{*}, A^{*}\right)$ and $F^{*}<1$. Its characteristic equation at the positive steady state takes the form

$$
\lambda^{2}+a \lambda+b \lambda e^{-\lambda \tau}+c+d e^{-\lambda \tau}=0,
$$

where

$$
\begin{array}{ll}
a=m_{J}+m_{A}+a_{J} s, & b=b(\tau)=\left(a_{A}-a_{J}\right) s e^{-m_{J} \tau}, \\
c=m_{A} m_{J}+a_{J} m_{A} s, & d=d(\tau)=\left(a_{A} m_{J}-a_{J} m_{A}\right) s e^{-m_{J} \tau} .
\end{array}
$$

Clearly, $c+d>0$, which implies $\lambda=0$ can never be a root of (4.28). When $\tau=$ $0,(4.28)$ reduces to

$$
\lambda^{2}+(a+b) \lambda+c+d=0,
$$

which has roots with negative real parts, implying that $\left(J^{*}, A^{*}\right)$ is locally asymptotically stable when $\tau=0$.

In Figure 3, we plot the graph of the map $S_{0}^{+}(\tau)$ versus $\tau$ in the interval $I=\left[0, \tau_{1}\right)$ for the set of parameters satisfying

$$
a_{A}=1, \quad m_{A}=1, \quad a_{J}=0.1, \quad m_{J}=1, \quad s=10,
$$


with $\tau_{1}=1.49$. It can be shown that for this set of parameters, $\omega_{-}$is not feasible for $\tau \in I$. The graph of $S_{0}^{+}(\tau)$ versus $\tau$ in Figure 3 shows that $S_{0}^{+}(\tau)$ has two zeros, the first at the value $\tau_{01}=0.29$, the second at the value $\tau_{02}=1.14$, whereas $S_{1}^{+}(\tau)<0$ on $I$. According to Theorem 4.1 and Remark 4.1, we see that at $\tau_{01}$ a stability switch occurs toward instability whereas at $\tau_{02}$ the stability switch occurs toward stability. Again, we see that intermediate delays show a destabilizing effect on the steady state, whereas large delays have a stabilizing one. For $\tau \in\left(\tau_{01}, \tau_{02}\right)$, the steady state is unstable, whereas it is asymptotically stable for $0 \leq \tau<\tau_{01}$ and for any $\tau>\tau_{02}$. These results are in agreement with our computer simulations using XPP.

5. Discussion. It is well known that for nonlinear delay systems (including many neutral delay systems) the occurrence of characteristic roots crossing the imaginary axis from left to right as the result of changing certain parameters often ensures the emergence of nontrivial periodic solutions near the steady state as it becomes unstable (Hale and Verduyn Lunel [17]). For well-constructed population models, this scenario is to be expected. XPP simulation can easily confirm this.

We would like to stress here that the geometric criterion presented in the previous section may also be applicable to models with several discrete delays or distributed delays.

Consider, for an example, the following Lotka-Volterra predator prey model with two discrete delays:

$$
\left\{\begin{array}{l}
x^{\prime}(t)=x(t)\left[e_{1}-a_{1} x(t)-a_{2} y(t-\sigma)\right] \\
y^{\prime}(t)=y(t)\left[-e_{2}+a_{3} x(t-\tau)-a_{4} y(t)\right]
\end{array}\right.
$$

where all parameters are positive constants. Assume further that it has a positive steady state $E^{*}=\left(x^{*}, y^{*}\right)$. Let $r=\sigma / \tau$. The system can be reduced to the following one with dimensionless time $t / \tau$, which, for simplicity, we again denote by $t$ :

$$
\left\{\begin{array}{l}
x^{\prime}(t)=\tau x(t)\left[e_{1}-a_{1} x(t)-a_{2} y(t-r)\right] \\
y^{\prime}(t)=\tau y(t)\left[-e_{2}+a_{3} x(t-1)-a_{4} y(t)\right]
\end{array}\right.
$$

At $E^{*}$, the characteristic equation is

$$
\lambda^{2}+\lambda a^{*} \tau+b^{*} \tau^{2}+c^{*} \tau^{2} e^{-\lambda(r+1)}=0,
$$

where $a^{*}=a_{1} x^{*}+a_{4} y^{*}, b^{*}=a_{1} a_{4} x^{*} y^{*}, c^{*}=a_{2} a_{3} x^{*} y^{*}$. Since $\tau=\sigma / r$, we see that the characteristic equation takes the form

$$
\lambda^{2}+\lambda A(r, \sigma)+B(r, \sigma)+C(r, \sigma) e^{-\lambda(r+1)}=0,
$$

where $A(r, \sigma)=a^{*} \sigma / r, B(r, \sigma)=b^{*} \sigma^{2} / r^{2}, C(r, \sigma)=c^{*} \sigma^{2} / r^{2}$, and $r$ may vary in $\mathbf{R}_{+}$. Clearly, for each fixed value of delay $\sigma$, we have a characteristic equation with parameters dependent on $r$. Notice that all coefficients of (5.4) are positive, and hence all its real roots must be negative. Stability switching may occur when imaginary roots $\lambda=i \omega$ exist and cross the imaginary axis.

It is straightforward to find that $\omega$ must be the solution of

$$
F(\omega, r):=\omega^{4}+\omega^{2}\left(A^{2}(r)-2 B(r)\right)+B^{2}(r)-C^{2}(r) .
$$

(For simplicity, we drop the dependence of $\sigma$.) This gives

$$
\omega_{ \pm}^{2}=\frac{1}{2}\left\{2 B(r)-A^{2}(r) \pm\left[\left(2 B(r)-A^{2}(r)\right)^{2}+4\left(C^{2}(r)-B^{2}(r)\right)\right]^{1 / 2}\right\} .
$$


Let $\theta(r) \in[0,2 \pi]$ be the solution of

$$
\cos \theta(r)=\frac{\omega^{2}(r)-B(r)}{C(r)}, \quad \sin \theta(r)=\frac{\omega(r) A(r)}{C(r)} .
$$

The imaginary roots $\lambda= \pm i \omega, \omega(r)>0$ will appear at the $r$ values which are zeros $r^{*}$ of

$$
S_{n}(r)=r+1-\frac{\theta(r)+2 n \pi}{\omega(r)}, \quad n \in \mathbf{N}_{0} .
$$

Hence, once we know such $r^{*}$, we know $\tau^{*}=\sigma / r^{*}$. This will give us a pair of delay values $\left(\tau^{*}, \sigma\right)$ at which the stability switch may be possible when increasing the value of $r=\sigma / \tau$ while keeping $\sigma$ fixed. Of course, such analysis can be performed for each $\sigma$ for which the solutions (5.6) are feasible. A similar procedure can be applied to $\sigma$ while keeping $\tau$ fixed.

As an example for the applicability of our geometric criterion to models with distributed delays, we consider the following model of single species growth:

$$
x^{\prime}(t)=f\left(\int_{-\tau}^{0} e^{d s} x(t+s) d s\right)-g(x(t)),
$$

where $\tau$ is the maximum stage delay and $d$ is the through-stage death rate, both positive constants. The first term accounts for the births due to all the age groups and the second term represents the death rate. Typical assumptions on $f$ and $g$ are given in [20]. This model can also be viewed as a direct extension of the models studied in [10]. Assume that it admits a positive steady state of $x(t)=x^{*}$. Then

$$
f\left(\frac{1}{d}\left(1-e^{-d \tau}\right) x^{*}\right)=g\left(x^{*}\right) .
$$

Let

$$
a:=f^{\prime}\left(\frac{1}{d}\left(1-e^{-d \tau}\right) x^{*}\right), \quad b:=g^{\prime}\left(x^{*}\right) .
$$

Then the linearized equation of (5.9) takes the form

$$
u^{\prime}(t)=a \int_{-\tau}^{0} e^{d s} u(t+s) d s-b u(t) .
$$

By differentiating the above equation one more time and making some simple substitution, we have

$$
u^{\prime \prime}(t)+(b+d) u^{\prime}(t)+(d b-a) u(t)+a e^{-d \tau} u(t-\tau)=0 .
$$

Clearly, this is a special case of (4.1) and our criteria are applicable.

There are several factors that may have contributed to the current prevalence of models containing only delay independent parameters. These include the following: (1) the authors fail to recognize the need to have some of the parameters become delay dependent; (2) the authors think that delay independent parameters can provide a good enough description or approximation of the dynamics; (3) there is simply a lack 
of mathematical results and methods to deal with models involving delay dependent parameters.

To see why it is easy to overlook the need of introducing delay dependent parameters in a model, let us consider the well-studied Nicholson's blowfly model proposed in $[16]$ :

$$
N^{\prime}=R-D=R(N(t-\tau))-\delta N=p N(t-\tau) e^{-N(t-\tau)}-\delta N
$$

where $N$ is the sexually mature adult blowfly population density, $\delta$ is its individual death rate, and $R$ is the recruitment rate. In arriving at the above form of $R$, Gurney, Blythe, and Nisbet [16] argued that (1) the rate at which eggs are produced depends only on the current size of adult population, (2) each egg takes about $\tau$ units of time to become a sexually mature adult, and (3) the probability of a given egg maturing into a viable adult depends only on the number of competitors of the same age. However, since an individual larva will die at a constant or average rate of $d$, the through-stage survival rate for a larva to adulthood is $e^{-d \tau}$. Thus a more plausible model should take the form (Cooke et al. [10])

$$
N^{\prime}=e^{-d \tau} R(N(t-\tau))-\delta N=p e^{-d \tau} N(t-\tau) \exp \{-N(t-\tau)\}-\delta N
$$

Now, this equation has a parameter $e^{-d \tau}$ that depends on the time delay $\tau$.

To see the importance of introducing delay dependent parameters, we again use models (5.12) and (5.13). It is well known [21] that for (5.12), if a positive steady state exists and this steady state is unstable for $\tau=\tau_{0}$, then it remains unstable for $\tau>\tau_{0}$. That is, a large time delay plays a destabilizing role. However, for model (5.13), one sees the opposite [10]. What is often observed (which can be easily verified by XPP) is that there are two threshold values $0<\tau_{0}<\tau_{1}$ such that the positive steady state is unstable only when $\tau \in\left[\tau_{0}, \tau_{1}\right]$. Dramatic differences in dynamics provided by models with delay dependent parameters and models with delay independent parameters like that above seem to be the rule rather than the exception. It is also worth mentioning here that applying existing criteria designed for models with delay independent parameters can lead to speculative or even erroneous statements.

Acknowledgments. The authors would like to thank the two referees for their constructive comments and suggestions.

\section{REFERENCES}

[1] W. G. Aiello And H. I. Freedman, A time-delay model of single-species growth with stage structure, Math. Biosci., 101 (1990), pp. 139-153.

[2] W. G. Aiello, H. I. Freedman, and J. Wu, Analysis of a model representing stage-structured population growth with state-dependent time delay, SIAM J. Appl. Math., 52 (1992), pp. 855-869.

[3] J. R. Bence AND R. M. NisBet, Space-limited recruitment in open systems: The importance of time delays, Ecology, 70 (1989), pp. 1434-1441.

[4] E. Beretta And Y. KuAng, Modeling and analysis of a marine bacteriophage infection with latency period, Nonlinear Anal. Real World Appl., 2 (2001), pp. 35-74.

[5] S. P. Blythe, R. M. Nisbet, W. S. C. Gurney, and N. MacDonald, Stability switches in distributed delay models, J. Math. Anal. Appl., 109 (1985), pp. 388-396.

[6] F. G. Boese, Stability with respect to the delay: On a paper of K. L. Cooke and P. van den Driessche, J. Math. Anal. Appl., 228 (1998), pp. 293-321.

[7] K. L. Cooke and Z. Grossman, Discrete delay, distributed delay and stability switches, J. Math. Anal. Appl., 86 (1982), pp. 592-627.

[8] K. L. CoOke And P. VAn Den DRIEsche, On zeros of some transcendental equations, Funkcial. Ekvac., 29 (1986), pp. 77-90. 
[9] K. L. Cooke and P. VAn Den Driesche, Analysis of an SEIRS epidemic model with two delays, J. Math. Biol., 35 (1996), pp. 240-260.

[10] K. L. Cooke, P. VAn Den Driessche, and X. Zou, Interaction of maturation delay and nonlinear birth in population and epidemic models, J. Math. Biol., 39 (1999), pp. 332-352.

[11] J. M. Cushing, Integrodifferential Equations and Delay Models in Population Dynamics, Lecture Notes in Biomath. 20, Springer-Verlag, Berlin, New York, 1977.

[12] J. M. Cushing, An Introduction to Structured Population Dynamics, CBMS-NSF Regional Conf. Ser. Appl. Math. 71, SIAM, Philadelphia, PA, 1998.

[13] B. Ermentrout, XPPAUT Ver. 5.5, University of Pittsburgh, Pittsburgh, PA; available online at http://www.math.pitt.edu/ bard/xpp/xpp.html.

[14] H. I. Freedman And Y. KuAng, Stability switches in linear scalar neutral delay equations, Funkcial. Ekvac., 34 (1991), pp. 187-209.

[15] H. I. Freedman and V. S. H. RaO, The tradeoff between mutual interference and time lags in predator-prey systems, Math. Biosci., 45 (1983), pp. 991-1004.

[16] W. S. C. Gurney, S. P. Blythe, And R. M. Nisbet, Nicholson's blowflies revisited, Nature, 287 (1980), pp. 17-21.

[17] J. Hale And S. M. Verduyn Lunel, Introduction to Functional Differential Equations, Springer-Verlag, New York, 1993.

[18] V. A. A. Jansen, R. M. Nisbet, And W. S. C. Gurney, Generation cycles in stage structured populations, Bull. Math. Biol., 52 (1990), pp. 375-396.

[19] V. L. Kocic and G. Ladas, Global Behavior of Nonlinear Difference Equations of Higher Order with Applications, Kluwer, Dordrecht, 1993.

[20] Y. KuAnG, Global attractivity and periodic solutions in delay differential equations related to models of physiology and population biology, Japan J. Indust. Appl. Math., 9 (1992), pp. 205-238.

[21] Y. Kuang, Delay Differential Equations with Applications in Population Dynamics, Academic Press, Boston, 1993.

[22] Y. Kuang And J. W.-H. So, Analysis of a delayed two-stage population model with spacelimited recruitment, SIAM J. Appl. Math., 55 (1995), pp. 1675-1696.

[23] R. M. Nisbet, W. S. C. Gurney, and J. A. J. Metz, Stage structure models applied in evolutionary ecology, Biomathematics, 18 (1989), pp. 428-449.

[24] J. Roughgarden And Y. Iwasa, Dynamics of metapopulations with space-limited subpopulations, Theoret. Population Biol., 29 (1986), pp. 235-261.

[25] J. Roughgarden, Y. Iwasa, and C. Baxter, Demographic theory for an open marine population with space-limited recruitment, Ecology, 66 (1985), pp. 54-67. 